\title{
Verbe coranique et magie en terre d'Islam
}

Between al-Bûnî's Magical Formulae and al-Gazâlî's Islamic Prayers: Talismanic

Texts

\section{Pierre Lory}

\section{OpenEdition}

\section{Journals}

Édition électronique

URL : https://journals.openedition.org/span/1337

DOI : $10.4000 /$ span. 1337

ISSN : 2268-1558

Éditeur

École pratique des hautes études. Sciences humaines

Édition imprimée

Date de publication : 1 novembre 1993

Pagination : 173-186

ISSN : 0294-7080

\section{Référence électronique}

Pierre Lory, "Verbe coranique et magie en terre d'Islam », Systèmes de pensée en Afrique noire [En ligne], 12 | 1993, mis en ligne le 12 décembre 2013, consulté le 21 septembre 2021. URL : http:// journals.openedition.org/span/1337 ; DOI : https://doi.org/10.4000/span.1337 


\section{VERBE CORANIQUE ET MAGIE EN TERRE D'ISLAM}

\section{par}

\section{Pierre Lory}

De nombreuses formes de pensée magique de par le monde accordent au verbe, proféré ou écrit, une part essentielle dans la symbolique et dans le déroulement du rite. Il arrive très fréquemment que ce verbe ait déjà, dans la cosmogonie, une fonction instauratrice: c'est par la parole que le Dieu origine de l'univers produit à l'existence les choses et les individus, qu'il les ordonne et les dirige. Par cette imitation sacrée qui fonde souvent la pratique culturelle et/ou la magie, l'homme de connaissance et de pouvoir peut à son tour manier cette parole instauratrice, dont la fonction n'est pas uniquement de fournir une information comme le fait le discours usuel, mais d'exercer une action sur le déroulent des processus vitaux.

Ces considérations renvoient un écho tout particulier en climat culturel islamique, où la Parole joue un rôle religieux de premier plan. Le Coran est reçu comme un message divin "dicté" pour ainsi dire au prophète Muhammad par l'Ange, et dont chacun des mots, des phrases et des versets a été voulu et construit par l'intention divine. En récitant à son tour le texte du Coran, le simple croyant ré-actualise la descente du Verbe sur terre; il prend en quelque sorte la position de l'ange de la révélation. Les mots qu'il articule ne sont pas assimilables à une

Fétiches II. Puissance des objets, charme des mots, Systèmes de pensée en Afrique noire, 12, 1993 
simple récitation liturgique, car ils sont tout chargés du pouvoir de l'énergie divine même qui les a proférés. Par cette récitation du Coran, le Musulman pratiquant s'approprie l'énergie divine, il se laisse compénétrer par son efficience surnaturelle. On comprendra sans peine que cette valeur quasi sacramentelle accordée à la lecture du Coran ait dès les premières générations de l'Islam glissé vers des pratiques plus utilitaires, et qu'on ait utilisé des versets à des fins de guérison ou de divination.

L'idée que nous voudrions avancer et illustrer ici est qu'en climat islamique, la parole n'est pas seulement un élément parmi d'autres dans les pratiques "occultes", mais qu'elle y exerce une fonction d'encadrement et de structuration de l'ensemble de la pensée et de l'agir magique. Il existe certes, comme partout dans le Proche-Orient antique et moderne, une magie sympathique où la pensée et les pratiques se meuvent selon des rapports d'analogie. De même, le rôle des considérations astrologiques est omniprésent dans la plupart des textes de quelque importance. Toutefois, il apparaît que la magie du verbe précède et informe ces autres formes de raisonnement. C'est ainsi que les signes du zodiaque, les planètes ou les maisons lunaires sont "marqués" par des lettres distribuées sur chaque zone de l'espace dont elles désignent les qualités spécifiques. En effet, elles ne représentent pas seulement des signes, des reperes pour l'action céleste qui est désignée, mais sont des matrices de ces actions en quelque sorte, qui sont ontologiquement supérieurs aux astres. La tradition soufie y voit souvent des hiérarchies supérieures d'anges.

Cette préminence de la magie littérale sur les autres formes d'action occulte est surtout sensible dans les textes plus tardifs. Les premiers grands traités de la littérature magique arabe (comme l'cuure de Ibn Wahshiyya, ou le Ghâyat al-hakîm du pseudo-Majrîtî) dépendaient encore notablement de modèles antiques, et faisaient une part plus importante aux forces astrales ou aux "propriétés intrinsèques" (khawâss) des substances. Les textes plus récents, et dont se servent le plus souvent les praticiens modernes des sciences occultes, se fondent par contre pour l'essentiel sur la composition de formules, de talismans, de rituels etc. à partir de noms divins, de certains versets, de certaines lettres tirés du Coran; comme si toute magie dans le monde 
représentait en quelque sorte un prolongement, une réverbération de la parole divine adressée aux terriens. Nous référerons ici plus particulièrement au Shams al-ma'ârif de Bûnî (13e siècle), et au Shumûs al-anwâr d'Ibn al-Hâjj Tilimsânî (14e siècle) ${ }^{1}$.

\section{La grammaire cosmique}

Le nom d'une chose ou d'une personne est considéré, dans nombre de systèmes magiques, comme l'expression de son essence même. C'est également le cas pour nos textes de magie musulmane. Mais celle-ci insère cette croyance dans une vision sous-jacente plus générale, à savoir que l'ensemble des lois régissant l'univers obéissent à une sorte de syntaxe universelle, comprenant ses noms (les essences), ses adjectifs (les accidents), ses verbes (les processus de transformation). Or cette parole cosmique est parallèle à la parole humaine. Pour présenter les choses autrement, on pourrait dire que la langue humaine (il s'agit ici bien sûr de l'arabe) est un reflet du même ordre, de la même composition, de la même sagesse divine qui structure le monde entier. Ce qui engendre deux conséquences. D'une part, cela implique que celui qui connaît les secrets de la langue humaine pénètre dans les secrets les plus profonds de la création surtout si sa méditation porte sur la parole la plus pure, la plus divine qui puisse s'écouter sur terre, à savoir le Coran. D'autre part, cela suggère que I'homme connaissant la structure intime de cette langue, ayant compris que chaque lettre est, à son plus haut degré, un ange, se trouve par le fait même investi d'un pouvoir vaste (Sh. $M .: 14,63,75$ ).

Commençons par la définition de cette métalangue magique. Comment l'aborder? Il n'existe bien sûr pas d'enseignement en tant que tel. La tradition soufie orale et les quelques textes que nous possédons font état de l'explication que des maîtres ont fourni sur tel ou tel point particulier; mais il semble surtout que la "science des lettres"

\footnotetext{
1 Shams al-ma'ârif wa-latâ 'if al-'awârif, Matba'a Mustafâ Muhammad, Le Caire, s.d., ici Sh. M. ; et Shumûs al-anwâr wa-kunûz al-asrâr, Al-Maktaba al-falakiyya al'ilmiyya, Beyrouth, s.d., ici Sh. A.
} 
s'acquière par de longues méditations individuelles, suscitant la germination progressive de significations ésotériques dans l'esprit du méditant qui se présentent alors à lui comme autant d'inspirations, d'illuminations intérieures accompagnées ou non de visualisations ${ }^{2}$.

Mais ce caractère inspiré et assez arbitraire de l'apprentissage de la science des lettres n'a pas empêché cette dernière de se doter d'un certain nombre de principes et de règles stables. Le monde a selon nos textes été créé à partir des 28 lettres de l'alphabet arabe, qui ont composé les 99 Beaux Noms de Dieu (qui sont les matrices de tous les êtres créśs), puis se sont réparties dans l'ensemble du cosmos. Chaque constellation du Zodiaque, chaque corps céleste, chaque heure du nycthémère se trouve affecté d'une ou plusieurs lettres qui sont ses "patrons", son ange recteur. Réparties selon les Qualités Elémentaires (lettres chaudes, seches, froides, humides), les lettres structurent également les lois physiques du monde sublunaire. Il n'est pas un aspect de la vie matérielle ou psychique de chaque être humain qui ne puisse être traduit selon cet alphabet cosmique, ce repérage servant bien entendu aussi de diagnostic (Sh. M.: 5 \& s., Sh. A.: 77 et Lory, 1989b).

La lecture attentive du Coran vient enrichir constamment ce type de spéculation. On tiendra compte du rôle les lettres lumineuses (lettres séparées mises en exergue de certaines sourates) opposées aux autres, "ténébreuses". On relèvera quelles lettres font partie de la première sourate, la Fâtiha, et quelles autres en sont absentes. Partant de l'idée plus ou moins exprimée que le verbe coranique attire la présence des anges, que ses sourates, versets ou lettres sont elles-mêmes, au plus haut niveau de l'être, des entités angéliques (cf. Ibn Arabi, 1988: 454 \& s., et $S h$. M.: 14), la récitation répétée ou la composition de talismans cherchera à comprendre et maîtriser cet influx d'en haut. La

2 Ibn Arabi, dans le 2ème chapitre des Futûhât al-Makkiyya consacré à la science cachée des lettres, signale à plusieurs reprises que seul le dévoilement intuitif (kashf) et non le raisonnement discursif, permet d'accéder aux secrets métaphysiques de l'alphabet. Cf. à ce sujet la traduction française des principaux passages sur cette science par Gril, dans Ibn Arabi 1988: 382-487. Non moins frappant dans le domaine de cette science illuminative du langage est le cas du soufi marocain 'Abd al-'Aziz alDabbâgh, évoqué infra, note 4. 
méditation prendra appui ici sur des spéculations arithmologiques parfois extrêmement complexes cherchant à traduire les lettres considérées en nombres (le nombre étant l' "esprit" de la lettre), puis à ramener ces nombres à des noms prononçables. A chaque fois, l'intention sera voisine: puisque tout ce qui advient dans notre monde sensible est le résultat d'interactions survenant dans les mondes supérieurs, l'activité magique cherchera à intervenir directement dans ces zones subtiles "avant" la manifestation de l'événement attendu ou redouté (Sh. M.: 57-59).

La langue arabe devient ici un support matériel concret pour la découverte et l'appropriation de niveaux de langage supérieurs. La compréhension et le maniement de la "langue des anges" est certes un domaine bien trop insaisissable pour qu'on puisse en parler avec précision. Nos textes y font allusion parfois en appelant cet idiome surnaturel al-suryâniyya, le syriaque. Il ne s'agit pas ici bien sûr de la langue araméenne occidentale désignée usuellement par ce terme, mais d'un mode de communication angélique qui se manifeste parfois dans les hadra soufies sous forme de phénomènes de glossolalie ${ }^{3}$. Nos auteurs se bornent à nous signaler à ce sujet qu'en suryâniyya, chaque lettre prise isolément est signifiante, porteuse du sens matriciel qu'elle diffuse dans les mondes inférieurs. Le Coran lui-même peut donc être "traduit" en suryâniyya, ce dont certains auteurs fort différents comme Bûnî en magie et 'Abd al-Azîz al-Dabbâgh dans le domaine de la mystique nous fournissent des échantillons ${ }^{4}$. Il s'agit bien sûr à chaque fois d'inspirations individuelles, ou de données de tradition qui ne laissent aucune prise à l'induction pour un observateur extérieur.

Mais le sens supérieur de l'arabe coranique, comme celui de la langue des anges, releve en fait d'un autre ordre, celui du nombre. Le nombre, nous le disions plus haut, est l'esprit de la lettre, celle-ci

${ }^{3}$ C'est à dire dans des cas de "possession angélique", où une entité spirituelle se manifeste par l'intermédiaire d'un humain; pour le cas inverse, où un homme s'adresse aux êtres subtils par des invocations et des serments, cf. Sh. A.: 133.

${ }^{4}$ Cf. Bûnî, 1956: 91 sq.; Sh. M.: 32. Le cas de A.A. al-Dabbâgh (XVIle-XVIIle siècles) est plus riche d'implications spirituelles. Malheureusement, les passages où il aborde la question du langage suryânyyya (v. Ahmad ibn al-Mubârak, 1984, vol. 1, 342 et s.) n'ont pas encore fait l'objet d'une étude suivie. 
prenant la fonction du corps pour le nombre - corps subtil si elle est prononcée, corps physique si elle est écrite (Sh. M.: 6, 78). Nous retrouvons des intuitions de base du pythagorisme, du platonisme du Timée, de plusieurs spéculations gnostiques, et bien sûr de la kabbale hébraïque. L'important pour nous est de noter l'aspect profondément islamique de ces spéculations arithmoloqiques. Celles-ci se déroulent selon la valeur numérique des lettres arabes et selon des données coraniques: ainsi p. ex. le nombre des lettres de la basmala, des lettres isolées, etc. (Sh. M.: 31s, 39s \& 58). La dialectique du pair et de l'impair y est guidée par la théologie unitarienne de l'Islam classique (Sh. M.: 60, 76). L'origine comme la finalité de la science musulmane des lettres repose donc sur une démarche numérologique qui lui est propre.

\section{Le pouvoir des mots}

De l'évocation de cette "grammaire de l'univers", on peut donc inférer que la connaissance profonde, ésotérique du langage donne accès à la connaissance des secrets de la nature. C'est très exactement ce que s'était proposé d'accomplir et de décrire l'école d'alchimistes placée sous le patronage de Jâbir ibn Hayyân. Pour elle, l'analyse méthodique de la composition de chaque substance, de la nature de chaque lettre (chaude ou froide, humide ou sèche) permettait de déterminer avec précision la composition intime de chaque minéral ou substance organique 5 . Mais il y a plus, car la science des lettres ne se présente pas comme un simple savoir, elle entend conférer un pouvoir réel sur l'objet ou la personne nommée.

C'est en effet une croyance très répandue, peut-être même universelle, que la profération du nom peut exercer une influence sur le nommé, le nom donnant en quelque sorte l'essence, le germe ontologique de chaque existence particulière. La magie en terre d'Islam a

5 Sur cet aspect de l'œuvre de Jâbir, v. Paul Kraus, 1986: 223 s., et Pierre Lory, 1989a: $124 \mathrm{~s}$. 
beaucoup développé ce type de considération, qui fut appliqué dans des domaines variés, suivant des modalités très différenciées. Cette efficacité du nom a été invoquée jusque dans la médecine populaire telle qu'elle est rapportée par exemple par Suyûtî dans son Kitâb al-rahma fi al-tibb: pour soigner une fière, donc un excès de chaleur, on pourra avoir recours à un talisman ou à une formule jaculatoire où domineront les lettres froides. Dans un tout autre domaine, celui de la mystique du tasawwuf, le disciple reçoit un nom divin à réciter comme dhikr personnel, et le contenu même de ce nom (p. ex. yâ Latîf, "ô Bienveillant") est censé induire une transmutation progressive de la personne du récitant, qui se dépouille de ses qualités purement humaine pour s'assimiler la bienveillance même de Dieu (Sh. M.: 140).

Mais l'application la plus fréquente de la puissance du mot dans la magie islamique concerne en fait l'invocation des esprits. Ces derniers sont désignés par le terme assez général de rahâniyyât, qui peut se rapporter à des catégories d'entités assez différenciées, principalement aux différentes classes d'anges, lesquels sont également appelés 'ulwiyyât, les êtres d'en haut, car résidant dans les différentes sphères célestes ou plus haut encore, près du Trône divin (Sh. M.: 63; Sh. A.: 75 \& s., 179). Mais les invocations et talismans les plus usuels s'adressent plus simplement aux sufliyyât, terme désignant parfois des anges de rang inférieur, et le plus souvent les djinns, êtres vivant sur terre comme les hommes, et comme eux de vertu variable, potentiellement pécheurs sans être pour autant systématiquement malfaisants. Enfin, la parole magique peut également s'adresser aux shayâtîn, démons plus nettement maléfiques de la compagnie d'Iblîs; mais nos textes sont singulièrement discrets sur ce genre de pratiques glissant dans la magie noire la plus répréhensible au regard de la Loi.

C'est ici que l'efficacité accordée au nom se manifeste dans toute son ampleur. A lire les textes de Bûnî ou de Tilimsânî, on constate que le praticien qui accomplit son rituel magique dans les règles peut imposer sa volonté de façon pratiquement illimitée aux djinns: par la parole il les rend visibles, par la parole il les contraint, même contre leur gré, d'accomplir sa propre volonté sans contrepartie réelle de sa part. Ces djinns sont appelés souvent "serviteurs de (tel) Nom". Il suffit de connaître celui-ci et de savoir s'en servir pour obliger le djinn 
à partir (exorcismes, guérisons) ou au contraire à demeurer dans un endroit, à fournir des informations (cas de la divination), à accomplir des prodiges etc. Il peut s'agir d'un des 99 Beaux Noms de Dieu, mais aussi du nom propre d'un ange supérieur ou du djinn lui-même, ou encore d'une formule magique donnée telle quelle, dont le sens n'est pas explicité.

Les purifications rituelles et souvent les prières et les jeûnes sont requis, mais il semble toutefois que ce soit l'accomplissement correct de l'acte magique qui assure son efficacité, et que celle-ci ne soit donc directement liée ni au mérite d'une piété ou d'une vertu personnelle, ni à une sainteté particulière accordée par Dieu. Les actes évoqués dans nos manuels de magie sont d'ailleurs eux-mêmes souvent éloignés des préceptes fondamentaux de la religion: ainsi lorsqu'il s'agit de rendre malade son ennemi, voire de le faire mourir ${ }^{6}$. L'efficacité du rite ne dépend apparemment pas non plus d'un échange entre le magicien et le djinn, ce dernier devant obéir à l'ordre qui lui est impéré sans contrepartie apparente.

Or cette maîtrise conférée au magicien à l'égard des djinns, des sufliyyât, se retrouve en fait également dans ses rapports avec les anges et les entités supérieures. Certes, les invocations sont plus respectueuses, les rites plus exigeants notamment dans le respect des rythmes astrologiques. Mais nulle part n'apparaît l'éventualité qu'un ange puisse refuser d'accomplir l'injonction qui lui est adressée, fût-il l'un des quatre archanges porteurs du Trône.

L'assimilation la plus complète entre sainteté et compétence en magie littérale apparaît dans l'idée que, par la connaissance de formules de prière appropriées, le praticien en magie peut s'attirer les faveurs du Dîwân al-Sâlihîn. Il s'agit de l'assemblée des plus grands saints des temps passés, présidée par le prophète Muhammad luimême, et qui joue apparemment un rôle de relais de l'initiative divine, intervenant dans les affaires terrestres pour guider ou guérir, avertir ou châtier, ou simplement pour intercéder auprès de Dieu. Certaines

${ }^{6}$ La puissance conférée sur les djinns en particulier est inconditionnelle (Sh.A., 6

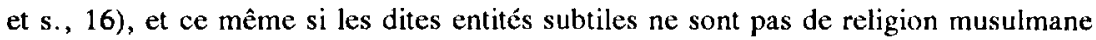
(Sh.A. ,129). 
formules permettent d'acquérir leur soutien, leur science ou leur pouvoir, voire même de devenir membre de leur assemblée (Sh.A.: 9, 14-15, 65). Le magicien obtient donc pour lui la capacité de participer aux plus hautes instances décidant du sort de l'humanité.

Il va en fait même jusqu'à suggérer implicitement qu'il se trouve investi d'une véritable puissance divine, puisque c'est au(x) Nom(s) de Dieu qu'il convoque et asservit les djinns, qu'il obtient le concours des anges et des saints (Sh.A.: 16). Son attitude n'est donc pas directement assimilable à de l'impiété ou de la mégalomanie, puisqu'il entend simplement faire usage de moyens d'agir qui lui ont été accordés par le Créateur, prolongeant la volonté de Celui-ci, sans s'y opposer ou lui substituer la sienne propre. On aurait tort, nous semble-t-il, de juger cette attitude selon une perspective théologique et juridique. Nous sommes cependant conduits à regarder d'un peu plus près les rapports entre ces pratiques magiques et les comportements et idées plus strictement religieux en Islam.

\section{Mystique et magie}

Si les théologiens musulmans ont pour la plupart récusé la pratique de la magie, la jugeant au mieux comme inutile, au pire comme une blâmable impiété, passible dans certains cas de la peine capitale, la réalité sociale est en fait beaucoup plus nuancée. Nous ne faisons pas ici seulement allusion au phénomène du "maraboutisme" tel qu'il a été décrit au Maghreb et en Afrique soudanaise ces derniers siècles, car si l'on interroge les premiers documents qui nous ont fait connaître l'émergence du soufisme en Islam, on s'aperçoit combien la notion de "sainteté" (gardons ce pis-aller pour traduire walâya) a été dès le départ liée à des manifestations préternaturelles de façon très étroite ${ }^{7}$. Certes les hagiographes les plus soucieux de démontrer le caractère

\footnotetext{
${ }^{7}$ Sans même parler des pouvoirs surnaturels qui étaient attribués, de son vivant, à la personne même du prophète Muhammad, il semble que les imâms chiites aient été l'objet de ce type de croyance de la part de certains de leurs partisans dès le premier siècle de l'Hégire.
} 
orthodoxe et honorable du tasawwuf ont souligné qu'il s'agissait de pures grâces divines (karâmât). Mais il n'est pas sûr du tout que le public non soufi ait perçu les choses de cette façon, et des personnages historiquement déjà bien connus comme Dhû al-Nûn Misrî ou Hallâj ont été accusés par leurs adversaires de magie vulgaire et de sorcellerie, tandis que leurs admirateurs voyaient en eux les dépositaires de sciences ésotériques d'origine divine.

L'opinion du théologien, comme celle de l'historien de la pensée, se fonde le plus souvent sur un jugement normatif. Chaque discours, même religieux ou magique, étant repéré, classé, devait se rapporter à une zone bien précise de la réalité sociale ou du psychisme. On a de ce fait souvent abouti à exclure l'une de l'autre des attitudes logiquement incompatible, mais pratiquement assumées et vécues simultanément, comme p. ex. la foi musulmane et les pratiques occultes. La coalescence entre soufisme et pouvoir magique apparaît plus clairement, me semble-t-il, si l'on prend en compte les données suivantes:

- D'abord, tous les "magiciens", auteurs ou consommateurs de textes occultistes de la période considérée (le bas Moyen-Age), ont certainement été des Musulmans et des Soufis complètement sincères. On ne distingue plus chez eux de relents de données païennes (comme chez Ibn Wahshiyya), et même l'astrologie se trouve comme "encadrée" par des considerations sur les 99 Beaux Noms de Dieu. Qu'ils aient été plus ou moins intéressés par l'argent ou le pouvoir est une autre question. Mais il me semble hors de doute qu'ils se voulaient, se pensaient Musulmans; plus encore, qu'ils se considéraient comme les dépositaires des plus hautes sciences dérivant de la révélation coranique, et d'un pouvoir prolongeant celui de Dieu ${ }^{8}$.

- Ensuite, il semble loisible d'attendre de l'adhésion au Coran des bienfaits surabondants, et ce non seulement dans l'Au-delà avec les récompenses promises aux croyants vertueux, mais également au cours de notre vie d'ici-bas. Le texte sacré lui-même le suggère. Le hadith est également prolixe pour décrire les avantages attachés au jeûne ei à

\footnotetext{
${ }^{8}$ Selon al-Bûnî, reprenant une sentence déjà très répandue avant lui, la basmala est au croyant ce que le fiat existenciateur (kun) est à Dieu (Sh.M., 186).
} 
la prière, avantages qui commencent dès le moment de la pratique. La mort physique marque certes un passage important, mais des son entrée en Islam, le croyant se trouve être l'objet de la miséricorde divine; les joies terrestres qu'il pourra connaître seront donc comme une anticipation du bonheur également sensible qu'il connaîtra dans le Jardin. Confectionner des talismans à l'aide de versets coraniques ou de noms divins n'a donc rien d'aberrant ou de blasphématoire: quel mal y aurait-il à puiser à cet océan de force et de grâces divines que manifeste le Verbe divin 9 ?

- Par ailleurs, l'accusation de culte rendu aux djinns, voire aux démons, qui est souvent lancée contre les praticiens des diverses sciences occultes, est peu justifiée, hormis sans doute certains cas de magie noire. Certes, le commerce avec les esprits (istihdâr al-rûhâniyyât) est l'un des aspects majeurs dans les pratiques de la magie musulmane. Mais il ne s'agit nullement d'un culte rendu à ces entités, ni non plus, a fortiori, une adoration concurrente de celle due à Dieu. Les rahâniyyât, nous l'avons vu, sont contraints d'obéir au magicien, non en vertu d'une autorité propre que posséderait celui-ci, mais à cause du pouvoir du nom divin ou du talisman en dérivant. C'est au nom de Dieu, par son pouvoir, que l'opération magique a lieu. Il y a donc là une différence essentielle avec la magie pré-islamique, où les djinns notamment étaient invoqués pour servir d'intermédiaires, voire d'intercesseurs entre les hommes et Dieu, et où ils occupaient par le fait même une fonction autonome et même une position de force par rapport à leurs "adorateurs"; c'est du moins ce qui ressort d'une série de citations coraniques et de mentions dans la litterature du hâdith et dans la Sîra (cf. à ce sujet: Lory, 1988: 86 \& suiv., Chelhod, 1964: 70 et $79 \&$ suiv., et Wellhausen, 1897: 149-172).

9 En effet, souligne Bûnî, la magie licite n'apporte que bénéfices dans l'au-delà pour le croyant (Sh.M.: 5). S'adresser aux forces astrales n'a rien d'impie, puisque celles-ci sont entièrement soumises à la volonté divine (ibid., 31). Quant aux vertus du Coran, elles sont infinies et s'étendent à tous les domaines (ibid., 186). 
Plus positivement, on peut considérer que la sainteté dans le soufisme "populaire" 10 est vue comme un accroissement simultané de la connaissance et du pouvoir surnaturel. Être saint, ce n'est pas seulement avoir acquis, par dévotion ou par grâce divine, un état de proximité purement mentale à Dieu. La conscience populaire n'imaginerait pas que cet état de proximité n'induise pas nécessairement des effets dans les mondes subtils et physiques, que la profusion de baraka qui en résulte ne se manifeste pas à l'extérieur par des guérisons, des prédictions, ou la simple diffusion d'un esprit de paix et de sérénité. Comment une personne récitant des invocations, coraniques ou non, des milliers de fois par jour, ne finirait-elle pas par aimanter autour de sa personne physique un faisceau de présences angéliques et de forces célestes? L'arrivée de phénomènes sur-naturels auprès des awliya', de leurs proches ou de leurs tombes devient dès lors un phénomène sinon "normal", du moins attendu. Le comportement même du saint n'est apparemment pas lié à un modèle unique. Il peut être ascète ou vivre dans le confort, ermite ou chef de guerre: le pouvoir qu'il transmet semble transcender sa propre personne et ses propres défauts apparents, voir ses échecs.

\section{Conclusion}

Il n'est pas toujours aisé pour autant d'évaluer le rapport exact entre attitude religieuse et pratique magique. Il semble en tout cas clair que la magie évolue dans une zone de la vie nettement profane: amour et guérison, argent et pouvoir sont les thèmes principaux qui lui sont attachés. Quant au salut de l'homme post mortem, il relève de l'exclusif vouloir divin: aucune invocation, aucun sacrifice, aucune interces-

${ }^{10}$ Nous plaçons cette expression entre guillemets, car l'observation précise montre bien qu'il n'existe aucune césure entre le soufisme "savant" (l'ouvre d'Ibn 'Arabi par exemple) et celui qui est pratiqué dans les milieux peu instruits, citadins ou ruraux. 
sion auprès des awliyấ ${ }^{\prime}$ ne vient déroger à ce principe ${ }^{11}$. Le saint peut guider dans une prise de décision, soulager telle ou telle souffrance, alléger un fardeau. Mais il ne peut pas forcer la mesure décidée par la Providence, même auprès de ses disciples sur la voie soufie qu'il s'est chargé de guider au seuil de la Présence divine. Son action est un simple prolongement du vouloir divin, mais qui rend ce dernier singulièrement plus concret pour les croyants ordinaires.

Au total, on constate que les pratiques magiques sont venues occuper l'espace précis qui avait été laissé vide par l'Islam littéraliste et le fiqh. Celui-ci prenait en charge tout ce qu'il est nécessaire de savoir et de faire en vue de l'agrément divin et de l'obtention de la récompense du Paradis, et ce par une Loi applicable uniformément pour chaque être humain. Les soucis et les détresses purement individuels, les besoins de soulagement immédiats, la nécessité de nourrir des espoirs simplement terrestres, tout ce qui pèse au jour le jour sur notre condition présente d'êtres humains, cela a été pris en charge par ces "hommes de pouvoir" que l'on hésite parfois à appeler des saints, mais qui sont venus apporter à de nombreuses couches de la société musulmane la dimension qui leur manquait: celle d'un surnaturel qui soit proche, domestique en quelque sorte, aussi riche en force et en potentiel que la vie sensible en est pauvre, un surnaturel qui s'adresse a chaque consultant, particulièrement, pour lui redire que sa vie est une promesse unique que Quelqu'un a prononcé devant Ses anges.

Pierre Lory

Ecole Pratique des Hautes Etudes

Section des Sciences Religieuses

\footnotetext{
${ }^{11}$ En fait, on peut trouver des invocations ou des talismans qui garantissent l'entrée dans le paradis, même au voleur ou à l'adultère (v. par ex. O. Depont et $X$. Coppolani, 1897: 140). Mais de telles assertions existent, sous forme de hadith appliquées à tel ou tel fragment du Coran, et doivent donc être situées dans un contexte religieux plus large que la simple pratique magique.
} 


\section{Références bibliographiques}

Ahmad ibn al-Mubârak

1984 Al-Ibrîz min kalâm sayyidî 'Abd al-'Azîz, Damas, ed. M.'A.alShammâ', vol. 1 .

Bûni, Ahmad ibn 'Alî

s.d. Shams al-ma'ârif wa-latâ'if al-'awârif, Matba'a Mustafâ Muhammad, Le Caire.

1956 Sharh al-Jaljalûtiyya al-kubrâ, dans Al-Usûl wa-dawâbit al-hikma, s.l., éd. A.A. Ibn Shaquîn.

Chelhod, Joseph

1964 Les structures du sacré chez les Arabes, Paris, Maisonneuve et Larose.

Depont, Oscar et Coppolani, Xavier

1897 Les confréries musulmanes, Alger.

Ibn Arabi

1988 Les Illuminations de La Mecque, Paris, Sindbad.

Ibn al-Hâjj Tilimsânâ

s.d. Shumûs al-anwâr wa-kunûz al-asrâr, Al-Maktaba al-falakiyya al-'ilmiyya, Beyrouth.

Kraus, Paul

1986 Jâbir ibn Hayyân - Contribution à l'histoire des idées scientifiques dans l'Islam, Paris, Les Belles Lettres.

Lory, Pierre

1988 "Sur la notion de Dieu dans la religion arabe anté-islamique", Cahiers d'Etudes Arabes, 2, p. 86 et suiv.

1989a Alchimie et mystique en terre d'Islam, Lagrasse, Verdier.

1989b "La magie des Lettres dans le Shams al-ma'ârif d'al-Bûnî", Bulletin d'Etudes Orientales, XXXIX-XL.

Wellhausen, J.

1897 Reste arabischen Heidentums, Berlin. 